\title{
CERN achievements in relativistic heavy ion collisions
}

\author{
Giuseppe Eugenio Bruno ${ }^{1, a}$ \\ ${ }^{1}$ Dipartimento Interateneo di Fisica 'M. Merlin' and Sezione INFN, Bari, Italy
}

\begin{abstract}
Twenty years after a Letter of Intent by the GSI and LBL groups for the "Study of particle production and target fragmentation in central ${ }^{20} \mathrm{Ne}$ on $\mathrm{Pb}$ reactions, at 12 $\mathrm{GeV}$ per nucleon energy of the CERN PS external beam" [1], based on the results found by the NA45/CERES, NA49, NA50, and WA97/NA57 experiments at the SPS, CERN announced compelling evidence for the formation of a new state of matter in heavyion collisions at CERN-SPS energies [2]. Some of the experiments were indeed the $2^{\text {nd }}$ or $3^{\text {rd }}$ generation successors of the apparatuses originally proposed by the GSI-LBL collaboration. Actually, the CERN ion program initiated at the SPS with the acceleration of oxygen ions at 60 and $200 \mathrm{GeV} /$ nucleon only in 1986, and continued with sulphur ions at $200 \mathrm{GeV} /$ nucleon up to 1993 . The rest is history: lead-ion beams at $160 \mathrm{GeV} /$ nucleon became available at the SPS in 1994; the LHC accelerated and collided lead beams at a center of mass energy per nucleon pair $\sqrt{s_{\mathrm{NN}}}=2.76 \mathrm{TeV}$ in 2010. Heavy ion physics is definitely in the future program of CERN: ALICE will operate a major upgrade of its detectors during the second long shutdown of the LHC, in 2018-2019, and the associated physics program will span the third and fourth LHC runs, till late 2020s.
\end{abstract}

\section{Foreword}

I was CERN summer student in 1998, and I have been asked to report on the CERN Achievements in Relativistic Heavy Ion Collisions, hence covering a period of time that dates back to 1980. Therefore, as a first task, I should define my role in this special session. I think that my role is simply that of historian ${ }^{1}$. Since this event has been held in Greece, where the father of history, Erodutus, conceived this art, I could empathize with the role easily.

\section{The dawn of relativistic heavy ion physics}

In October 1980, at the initiative of Rudolf Bock and Reinhard Stock, a Workshop on Future Relativistic Heavy-Ion Experiments took place at GSI Darmstadt [3]. The table of contents of the Workshop proceedings is reported in Fig. 1. This event is now considered as the first of Quark Matter Conferences, the major series of international meeting in the field of ultrarelativistic heavy-ion collisions.

\footnotetext{
a e-mail: Giuseppe.Bruno@cern.ch

${ }^{1}$ From a dictionary: "An expert in or student of history, especially that of a particular period, geographical region, or social phenomenon".
}

This is an Open Access article distributed under the terms of the Creative Commons Attribution License 4.0, which permits unrestricted use, distribution, and reproduction in any medium, provided the original work is properly cited. 

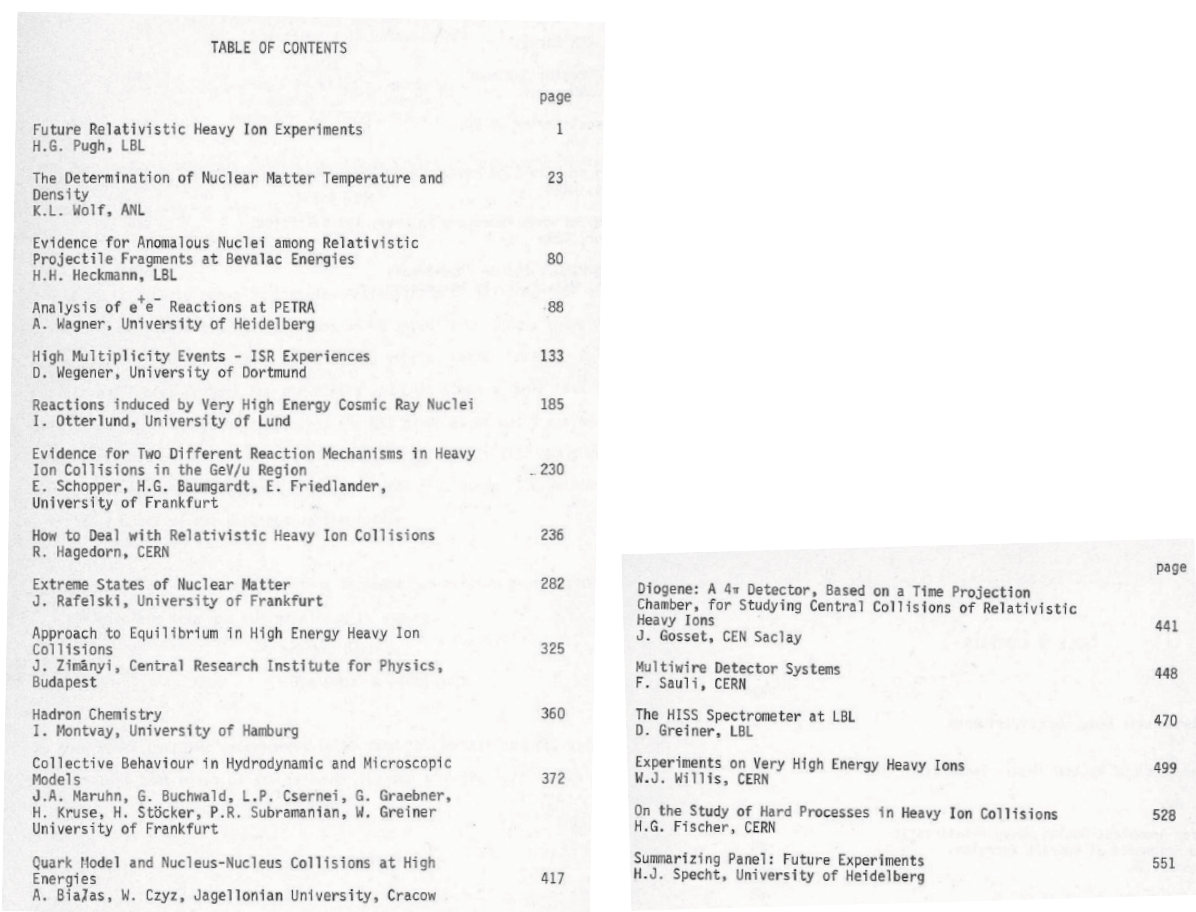

Figure 1. The table of contents of the Proceedings of the Workshop on "Future Relativistic Heavy-Ion Experiments", held in October 1980 at GSI.

But how did all that begin with? It is not possible to enucleate a single moment. In principle, one could go as back as to the Enrico Fermi and Isaak Pomeranchuk works [4, 5]. The introduction of statistical models in particle production dates back to 1950, well before the rise of the quark model in the 1960s. However, it is with Hagerdon's statistical bootstrap hypothesis [6, 7], in the second half of sixties, that the concept of a limiting temperature, $T_{\mathrm{c}}$, was proposed as the result of an exponentially increasing spectrum of hadronic states: hadronic matter cannot exist for $T>T_{\mathrm{c}}$.

The next two important steps forward date back to 1975: J.C. Collins and M.J.Perry speculated that superdense nuclear matter, at the center of a neutron star or in the early phases of the big-bang, consists of asymptotically free quarks rather than hadrons [8]. Independently, N. Cabibbo and G. Parisi demonstrated [9] that the exponentially increasing spectrum proposed by Hagedorn is not necessarily connected with a limiting temperature, since it must be present in any system that undergoes a $2^{\text {nd }}$ order phase transition, i.e. where thermodynamical quantities are singular at the transition temperature. The phase diagram of nuclear matter with a transition line appeared first in Cabibbo and Parisi's work. The existence of a different phase of the vacuum, in which quarks are not confined into hadrons, was suggested: when temperatures or densities become very high, strongly interacting quarks and gluons become free and give rise to a new, deconfined phase of matter, for which the term 'quark-gluon plasma' (QGP) was coined later on.

These findings brought to the idea of studying the collisions of heavy ions, accelerated at ultrarelativistic energies, with the hope to increase sufficiently the temperature and the density of nuclear matter. A few days after the Darmstadt workshop, a Letter of Intent for the "Study of particle produc- 

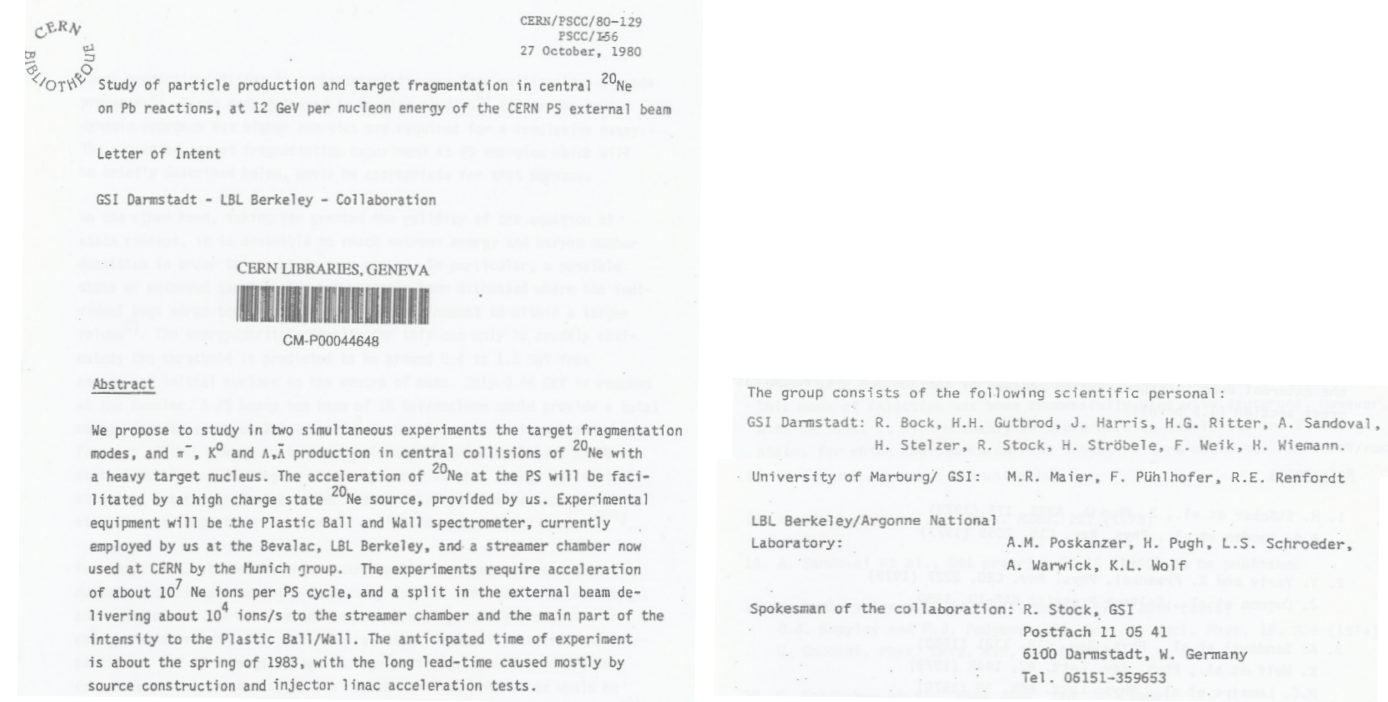

Figure 2. The first page of the 1980 Letter of Intent by the GSI-LBL collaboration and, right, the list of the authors of the proposal.

tion and target fragmentation in central ${ }^{20} \mathrm{Ne}$ on $\mathrm{Pb}$ reactions, at $12 \mathrm{GeV}$ per nucleon energy of the CERN Proton Synchroton external beam" was submitted by the GSI and LBL groups [1]. The first page of this seminal document, along with the list of authors of the proposal, is shown in Fig. 2.

\section{The past}

In 1980 the atmosphere was filled with enthusiasm and expectations. The possibility to produce in laboratory, albeit for a very short period, a deconfined state of quarks and gluons, the QGP, seemed to be at hand. However, some years had to pass before the first beams of oxygen ions at 60 and $200 \mathrm{GeV} /$ nucleon energy, values much larger than those initially envisaged, became available from the CERN Super Proton Synchrotron (SPS). At the time, in fact, the CERN priority was the Large Electron Positron (LEP) collider. Robert Klapisch, nominated Director of Research for all non-LEP activities in 1981, had the difficult mission to maintain a broad physics program, with reduced CERN investments.

Maurice Jacob, head of the CERN Theory Division from 1982 to 1988, played an important role in orchestrating interest among particle and nuclear physics groups to work together in this new field. In preparation of the possible SPS program, Maurice Jacob, together with Helmut Satz and William (Bill) J. Willis, organized the second Quark Matter meeting in Bielefeld in May 1982 [11]. The event brought together more than 100 participants from both sides of the Atlantic. At the initiative of Klapisch, a Workshop on the Future of Fixed Target Physics at CERN was held at CERN in December 1982; the group "Nuclear Beams and Targets" was convened by W.J. Willis and its work summarized by M. Albrow [12]. The greater opportunity in terms of both experimental capability and higher energy offered by CERN SPS became evident. As a result of all the mentioned efforts, a large fraction of the SPS community was encouraged to take an active interest in heavy-ion physics. 


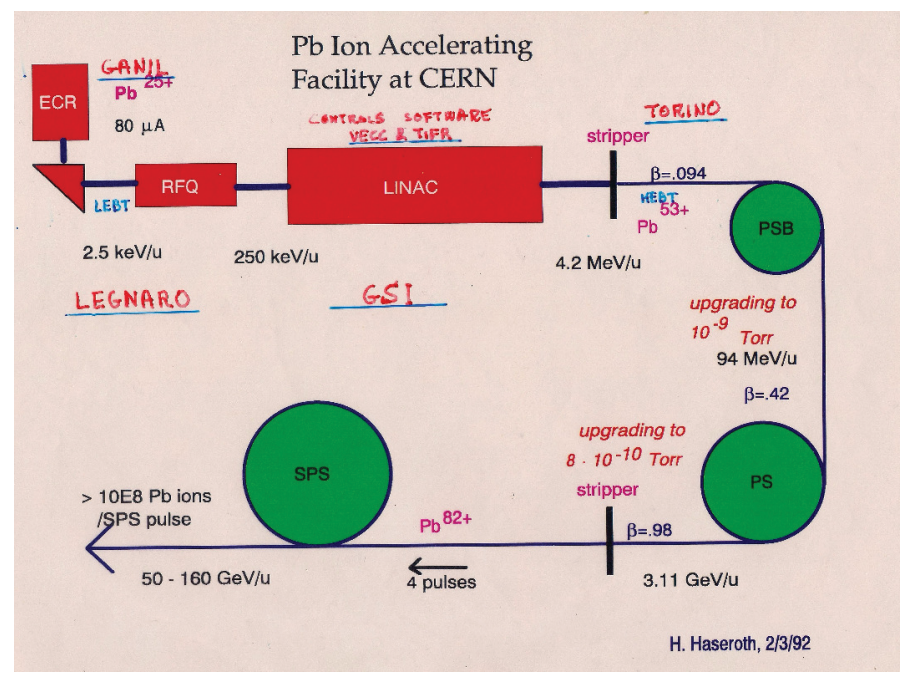

Figure 3. A schematic layout of the sequence of accelerators in the CERN heavy ion facility [10], with special emphasis on the tasks of the main collaborating institutions. The slide was prepared by Helmut Haseroth, the project leader of the CERN Heavy Ion Accelerating Facility.

The CERN heavy-ion facility, capable of accelerating ions up to sulphur at $200 \mathrm{GeV} /$ nucleon, was designed and constructed by several CERN member state laboratories, with contributions also of non-member states. A similar approach was used later in 1993 (see Fig. 3), when a major upgrade of the CERN ion facility was implemented to allow the acceleration of heavy ions, i.e. ${ }^{208} \mathrm{~Pb}[10]$.

In Autumn 1984, six new experiments were recommended by the SPSC (NA34/Helios, NA35, NA36, NA38, WA80, WA85); two of these (NA35 and WA80) were the direct offsprings of the 1980 Letter of Intent. The first apparatuses were assembled recycling many detectors and magnets from the previous experiments, while the second and third generation experiments required dedicated upgrades or new developments. A scheme of the approved ion experiments at the SPS with their temporal evolution is illustrated in Fig. 4. It would be interesting to go through the major technological developments introduced for these studies; for an overview of the CERN heavy-ion experiments active from 1986 to 2006, see for example [13]. I will focus, instead, on some of the main physics results from the SPS program.

Since the beginning, the characterization of the dynamical evolution of the collisions was studied with different approaches: the Hanbury-Brown and Twiss interferometry (see, e.g., [15]), the study of the transverse momentum distribution of identified particles, to determine the collective radial flow of the expanding fireball, or the combination of these two techniques [16], and the study of the azimuthal distribution of particles in the plane perpendicular to the beam direction ${ }^{2}$. For the last approach, it seems worth mentioning the results of NA49 [17], which demonstrated how shadowing by spectator matter cannot be at the origin of the observed elliptic flow at the SPS; it is instead caused by the pressure in the high density region created during the initial collision. In noncentral collisions the geometrical overlap region is anisotropic and this initial spatial asymmetry is converted via multiple collisions into an anisotropic momentum distribution of the produced particles.

\footnotetext{
${ }^{2}$ The second moment of the final state hadron azimuthal distribution is called elliptic flow.
} 


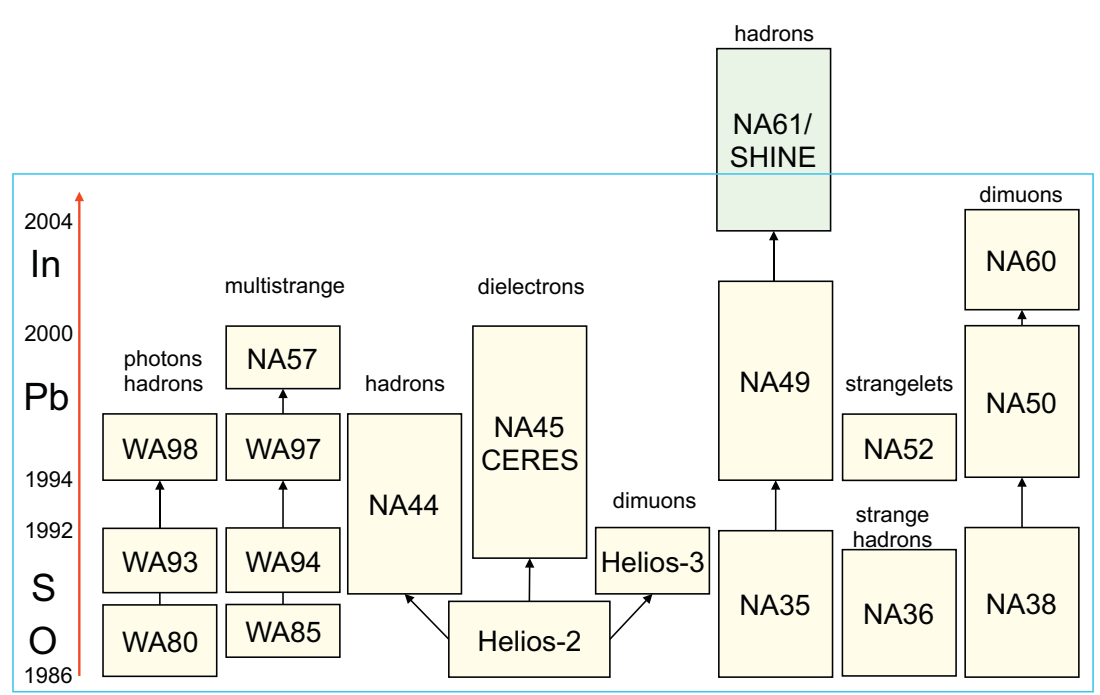

Figure 4. The ion experiments at the CERN SPS; all experiments are completed, except NA61/SHINE that is running [14].

The anomalous suppression of the $\mathrm{J} / \psi$ meson [18] and the strangeness enhancement [19] in nucleus-nucleus collisions were the two historical "signatures" of the QGP. The J/ $\psi$ formation would be suppressed due to the screening of the cc-potential in the presence of free color charges in the QGP. Conversely, if a QGP state were formed, an increased production of s and $\bar{s}$ quarks with respect

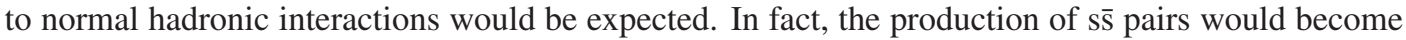

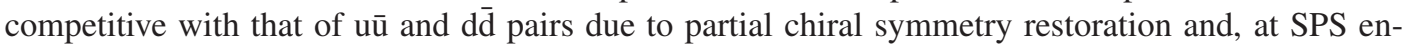
ergies, also because of the Pauli blocking of light quark production in a $\mathrm{d}$ and $\mathrm{u}$ rich environment.

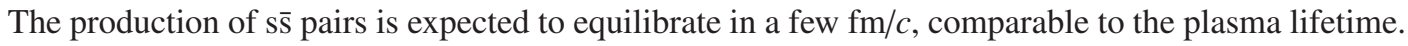
The net result, after statistical hadronization, would be an enhancement of strange and multistrange particle production in nucleus-nucleus interactions with respect to nucleon-nucleon interactions. The results for the anomalous $\mathrm{J} / \psi$ suppression measured by NA50 and the strangeness enhancement determined first by WA97, and later by other experiments, confirmed the predictions. The latest results obtained by NA50 [20] and NA57 [21] are shown in Fig. 5 and Fig. 6, respectively. In all plots the lines show expectations in the scenario of standard hadronic collisions.

Lepton pairs of low invariant mass were found very attractive for the investigation of high-energy nuclear collisions. In fact, in contrast to hadrons, they probe directly the entire space-time evolution of the expanding fireball formed in such a collision, escaping freely without final-state interactions. At low invariant masses, $m_{\mathrm{ll}}<1 \mathrm{GeV} / c^{2}$, thermal dilepton production is largely mediated by the light vector mesons $\rho, \omega$ and $\phi$. In particular the $\rho$, due to its strong coupling to the $\pi^{+} \pi^{-}$channel and its short lifetime, is subject to regeneration in the much longer-lived fireball. The $\rho$ meson is therefore the prime probe for in-medium modifications of hadron properties, including even the chiral symmetry restoration close to the QCD phase boundary. At intermediate masses, $m_{11}>1 \mathrm{GeV} / c^{2}$, where hadronic spectral functions become increasingly uniform, Planck-like thermal radiation is expected from both hadronic and partonic sources. Fig. 7 shows the dielectron invariant mass distributions measured by NA45 in different colliding systems [22-24]. While the more elementary collisions (p$\mathrm{Be}$ and $\mathrm{p}-\mathrm{Au}$ ) are reproduced within errors by final state Dalitz and direct decays of neutral mesons as 

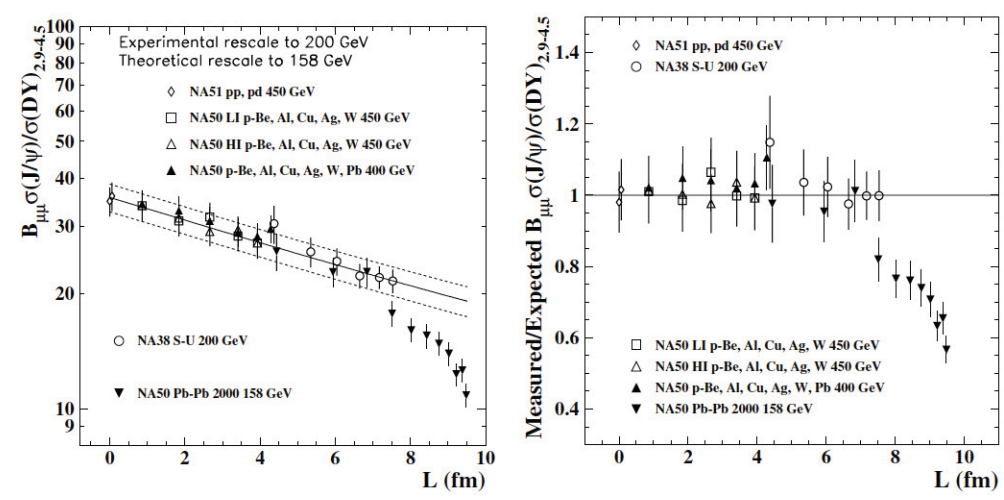

Figure 5. The $(\mathrm{J} / \psi) /$ Drell-Yan cross-sections ratio vs. $L$, the average length of nuclear matter traversed by the (pre-resonant) charmonium state, for several collision systems, compared to (left) and divided by (right) the normal nuclear absorption pattern [20].
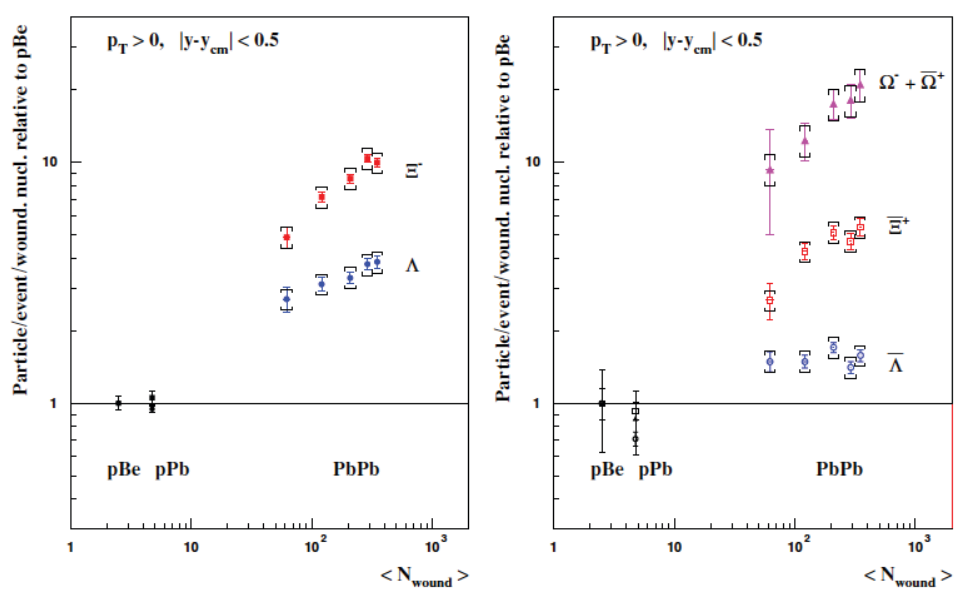

Figure 6. The hyperon enhancements as a function of the number of wounded nucleons measured by the NA57 experiment at central rapidity in $158 \mathrm{~A} \mathrm{GeV} / \mathrm{C} \mathrm{Pb}-\mathrm{Pb}$ collisions [21].

known from pp collisions, a substantial excess of dileptons above the known sources was found in the mass range above $250 \mathrm{MeV} / \mathrm{c}^{2}$ for $\mathrm{S}-\mathrm{Au}$ and $\mathrm{Pb}$-Au collisions, which is hard to be described without strong medium modifications of the $\rho$ meson.

The unprecedented precision of the NA60 experiment, which studied $158 \mathrm{~A} \mathrm{GeV/c} \mathrm{In-In} \mathrm{colli-}$ sions, allowed to set very stringent constraints on the models describing dilepton pair production; for a comprehensive discussion of the NA60 results one can refer to [25]. For the present purpose I will only focus on some important highlights. The left panel of Fig. 8 shows the inclusive invariant mass distribution of the excess dimuons, with all known sources subtracted (except for the $\rho$ ), integrated over $p_{\mathrm{T}}$, corrected for experimental acceptance and normalized absolutely to the charged-particle rapidity density [26]. A dropping of the $\rho$ nominal pole position, suggested by some models, was not 

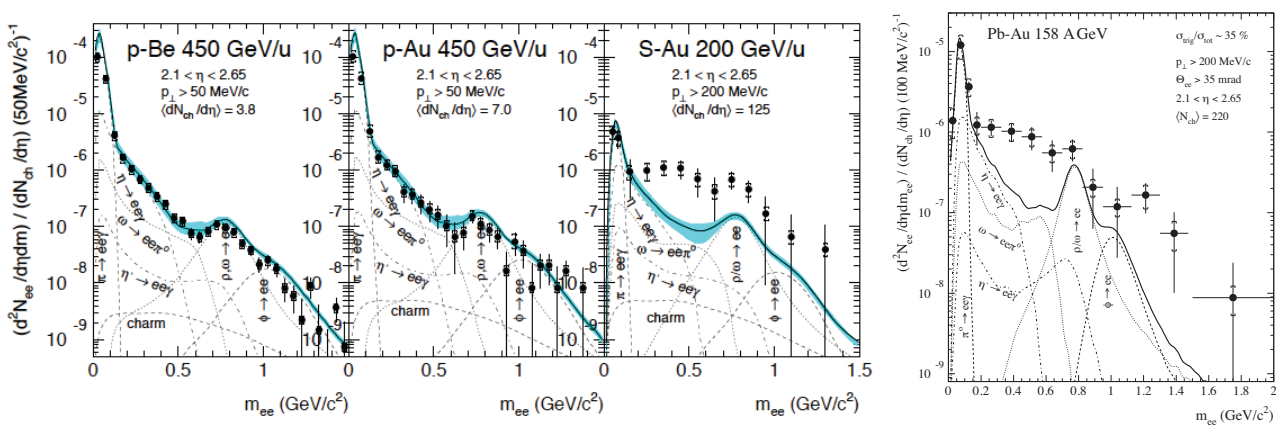

Figure 7. CERES inclusive $\mathrm{e}^{+} \mathrm{e}^{-}$mass distributions of $450 \mathrm{GeV}$ p-Be, $\mathrm{p}-\mathrm{Au}, 200 \mathrm{GeV} / N \mathrm{~S}-\mathrm{Au}$, and $158 \mathrm{GeV} / N$ $\mathrm{Pb}-\mathrm{Au}$ collisions [22-24]. The number of electron pairs per charged particle, in the acceptance and per event, is plotted. Contributions from various hadron decays, as expected from pp collisions, are shown together with their sum (thick line).
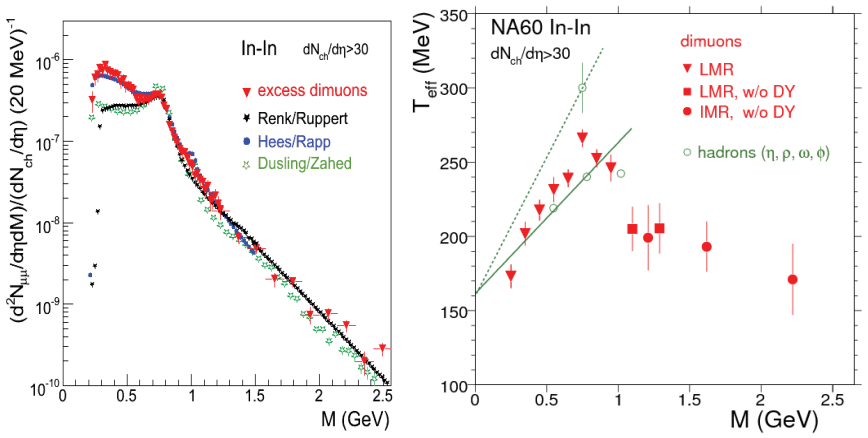

Figure 8. The up-to-date most precise measurement of dilepton production in heavy ion collisions, which has been performed by the NA60 collaboration [26] in 158 A GeV In-In collisions. Left panel: Acceptance-corrected invariant mass distribution of the excess dimuons, integrated over $p_{\mathrm{T}}$, compared with three different sets of thermal-model results in absolute terms. Right panel: Inverse slope parameter $T_{\text {eff }}$ of the acceptance-corrected $m_{\mathrm{T}}$ distributions vs. dimuon mass. Hadron results are shown for comparison.

observed while the width of the resonance was found significantly broader than in vacuum. The transverse mass $\left(m_{\mathrm{T}}=\sqrt{p_{\mathrm{T}}^{2}+m_{\mu \mu}^{2}}\right)$ distributions of the excess dimuons, obtained in slices of the invariant mass distribution, presented an exponential Boltzmann-like shape, whose inverse slope $\left(T_{\text {eff }}\right)$ is related to the temperature of the system (eventually blue-shifted by the collective expansion) at the different stages of its evolution. The right panel of Fig. 8 shows the dependence of $T_{\text {eff }}$ on $m_{\mu \mu}$, as compared to the inverse slope parameters extracted for hadrons. A natural interpretation of the sudden decline of $T_{\text {eff }}$ at masses larger than $1 \mathrm{GeV} / c^{2}$ implies dominantly early partonic processes like $q \bar{q} \rightarrow \mu^{+} \mu^{-}$for which collective flow has not yet built up. A fit to the momentum-integrated yield $\left(\mathrm{d} N / \mathrm{d} m_{\mu \mu}\right)$ of the excess dimuons above $m_{\mu \mu}=1 \mathrm{GeV} / c^{2}$ with a Planck-like shape can provide an absolute temperature $T$ (i.e. not affected by the collective expansion) of about $200 \mathrm{MeV}$. This temperature is well above the critical value $(170 \mathrm{MeV})$ of the QGP phase transition as predicted by lattice QCD calculations. 

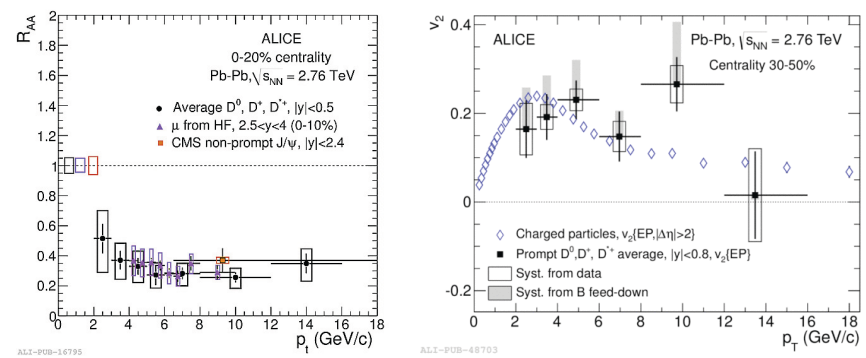

Figure 9. Left panel: Average $R_{\mathrm{AA}}$ of $\mathrm{D}$ mesons for the most central $20 \%$ of the inelastic $\mathrm{Pb}-\mathrm{Pb}$ cross section at $\sqrt{s_{\mathrm{NN}}}=2.76 \mathrm{TeV}$ [27] compared to the nuclear modification factors of muons from heavy flavor decays [28] and non-prompt $\mathrm{J} / \psi$ from $\mathrm{B}$ decays [29] for the same system. Right panel: Average of $\mathrm{D}^{0}, \mathrm{D}^{+}$, and $\mathrm{D}^{*+} v_{2}$ as a function of $p_{T}$ [30], compared to charged-particle $v_{2}$ [31].

In February 2000, at the start of the Relativistic Heavy Ion Collider program at the BNL, CERN announced compelling evidence for the formation of a new state of matter in heavy-ion collisions at CERN-SPS energies [2]. There was criticisms related to this announcement, of course. Today, when revising the SPS results, I think that a question should rise naturally: if it were not a Quark Gluon Plasma, then what could that system be?

But leaving the "discovery" aside, at the time the CERN challenge was on other fronts. In fact, while being at the peak of the SPS program, the same community had to face the LHC preparation: $\mathrm{R} \& \mathrm{D}$ activities, constructions of new detectors, physics simulations, physics data challenges. However, the most intriguing challenge was probably still a different one: coagulate several smaller groups into a single large new collaboration, ALICE. And this brings me to the present.

\section{The present}

LHC is the present, with a dedicated heavy ion experiment, ALICE, nicely complemented by the CMS and ATLAS heavy ion programs and, for $\mathrm{p}-\mathrm{Pb}$ collisions, by $\mathrm{LHCb}$ as well. Several interesting contributions about LHC experimental results have been presented at this Conference, but at least three measurements deserve a mention here as well.

- At LHC heavy flavour production can be measured with good precision, thanks to the large production cross sections of $c \bar{c}$ and $b \bar{b}$ pairs and to the presence of silicon vertex detectors in the experiments. In Fig. 9 the nuclear modification factor, defined as the yield per nucleon-nucleon collision in $\mathrm{Pb}-\mathrm{Pb}$ relative to that in $\mathrm{pp}$ and indicated with $R_{\mathrm{AA}}$, for exclusively reconstructed $\mathrm{D}$ mesons [27], for muons from heavy flavour decays [28] and for non-prompt $\mathbf{J} / \psi$ from beauty hadron decays [29], is shown as a function of $p_{\mathrm{t}}$. These results, together with the measurement of a sizeable elliptic flow for the $\mathrm{D}$ mesons in semi-central $\mathrm{Pb}-\mathrm{Pb}$ collisions [30], shown in the right panel of Fig. 9, pose stringent constraints on the values of the transport coefficients of the QGP.

- CMS has observed the predicted [32] sequential suppression of the $\Upsilon$ states in $\mathrm{Pb}-\mathrm{Pb}$ collisions [33]. Fig. 10 shows the dimuon invariant mass distributions measured in $\mathrm{Pb}-\mathrm{Pb}$ and $\mathrm{pp}$ collisions: the strongly bounded $\Upsilon(1 S)$ state survives in the hot medium, the loosely bounded $\Upsilon(2 S)$ is strongly suppressed, and the much weaker $\Upsilon(3 \mathrm{~S})$ disappears completely. In this case the "discovery" corresponds to the disappearance of peaks in an invariant mass distribution! 

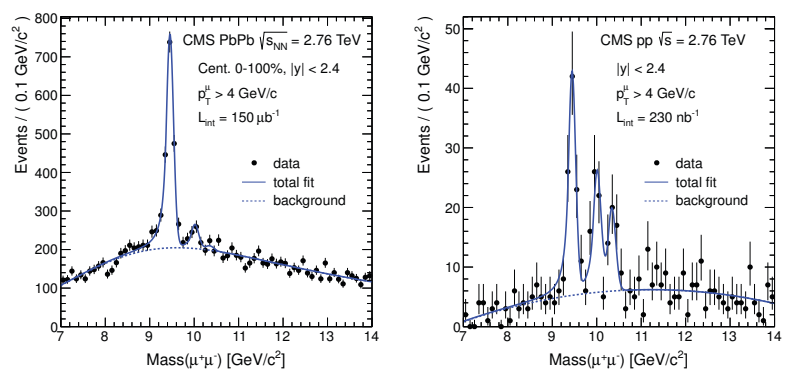

Figure 10. Dimuon invariant-mass distributions in $\mathrm{Pb}-\mathrm{Pb}$ (top) and pp (bottom) data at $\sqrt{s_{\mathrm{NN}}}=2.76 \mathrm{TeV}$ measured by CMS [33]. The solid (signal plus background) and dashed (background-only) curves show the results of the simultaneous fit to the two data sets.
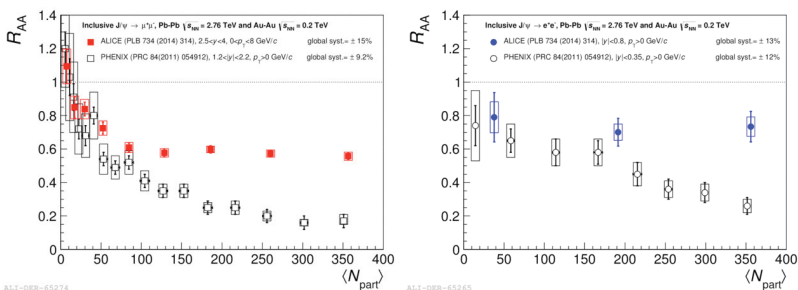

Figure 11. Centrality dependence of the nuclear modification factor, $R_{\mathrm{AA}}$, of inclusive $\mathrm{J} / \psi$ production in $\mathrm{Pb}-\mathrm{Pb}$ collisions at $\sqrt{s_{\mathrm{NN}}}=2.76 \mathrm{TeV}$, measured by ALICE at mid-rapidity (left) and at forward-rapidity (right) [34], compared to PHENIX result at RHIC in Au-Au collisions at $\sqrt{s_{\mathrm{NN}}}=0.2 \mathrm{TeV}$ [39].

- The $\mathrm{J} / \psi$ production at LHC shows a new regime: a smaller suppression is observed with respect to lower energies, as shown in Fig. 11, despite the higher energy density reached at LHC. When measured as a function of $p_{\mathrm{t}}$, the $R_{\mathrm{AA}}$ shows a strong dependence indicating that the smaller suppression measured at LHC is a low $p_{\mathrm{t}}$ effect. Such a new regime was indeed predicted [36-38] and it is ascribed to the recombination of ci pairs, abundantly produced at LHC energies, in the QGP or at the phase boundary. A first indication of a non null $\mathrm{J} / \psi$ elliptic flow [35] further corroborates this interpretation.

At present, heavy ion studies are being performed also at SPS, with the study of the properties of the transition between hadron gas and quark gluon plasma and the search of the critical point performed with a comprehensive scan in energy and size of colliding nuclei by the NA61/SHINE experiment [14].

\section{The future}

LHC is the future. With its second run an integrated luminosity of about $1 \mathrm{nb}^{-1}$ in $\mathrm{Pb}-\mathrm{Pb}$ collisions will be delivered to the experiments, a factor 10 larger than that of the first run. Then, in 2018 and 2019, during the second long shutdown of LHC, a major upgrade of the ALICE detectors will be operated [40], which would meet the expected increase in the luminosity of $\mathrm{Pb}-\mathrm{Pb}$ collisions, eventually reaching an interaction rate of about $50 \mathrm{kHz}$, i.e. instantaneous luminosity of $L_{\text {ist }}=6$. 


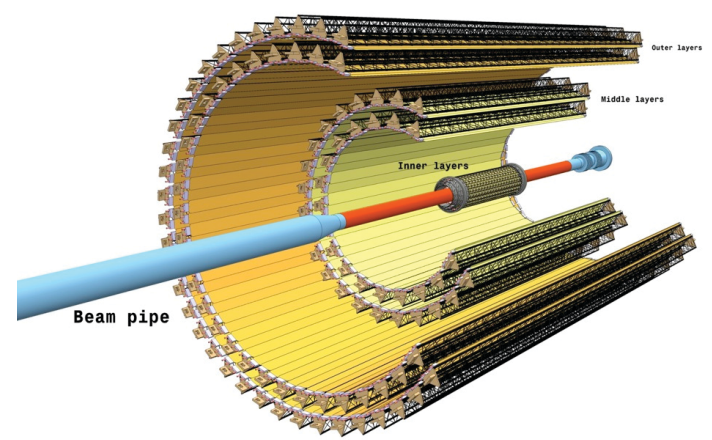

Figure 12. Layout of the new ALICE inner tracking system detector [41]

$10^{27} \mathrm{~cm}^{-2} \mathrm{~s}^{-1}$. With the high luminosity heavy ion program, which will span till late $2020 \mathrm{~s}$, the LHC experiments would be in a position to accumulate $10 \mathrm{nb}^{-1}$ of $\mathrm{Pb}-\mathrm{Pb}$ collisions on tape. Thanks to the new ALICE Inner Tracking System (ITS) [41], whose layout is shown in Fig. 12, the track position resolution at the primary vertex will be improved by a factor of 3 or larger with respect to the actual ALICE ITS detector. Given the accumulated statistics and the significantly improved resolution, the heavy flavour studies will definitely enter in the precision era.

The production of the top quark has never been observed in heavy ion collisions. Due to its large mass, differently from the other quarks the top quark does not hadronize before decaying, and it decays almost $100 \%$ of the time to $\mathrm{W}$ and $\mathrm{b}$ quark; therefore, by reconstructing the top decay products, one can study a bare quark and thus directly probe the earliest timescales of the interaction. With $10 \mathrm{nb}^{-1}$ of $\mathrm{Pb}-\mathrm{Pb}$ collisions, the CMS detector should be able to reconstruct about 500 top-anti-top pairs and study them for the first time in heavy ion collisions.

Other interesting projects are being considered at present. These include: (i) the mapping of the QCD phase diagram in the transition region, by studying the dimuon production in ion collisions at 20-160 A GeV/c at the CERN SPS, with an upgraded NA60 detector (NA60+) [42]; (ii) a study of the charmonium production at the SPS, including the $\chi_{c}$ meson, with a new apparatus equipped with a vertex/tracking detector, an electromagnetic calorimeter and a muon absorber/trigger system (CHIC) [43]; (iii) a multi-purpose fixed-target experiment using the proton and lead-ion beams of the LHC extracted by a bent crystal [44].

The acceleration of heavy ions at the Future Circular Collider (FCC) [45] can offer unprecedented physics opportunity covering the physics of quark gluon plasma, gluon saturation, photon-induced collisions, as well as connections with ultra-high-energy cosmic rays [46].

\section{Acknowledgement}

I would like to thank my friends and colleagues Roberta Arnaldi, Christoph Blume, Andrea Dainese, Marek Gaździcki, Bruno Ghidini, Emanuele Quercigh, Enrico Scomparin, Peter Seyboth, Gianluca Usai, for their help in the preparation of this contribution.

\section{References}

[1] GSI-LBL Collaboration, CERN/PSCC/80-129; PSCC/I-56 (1980). 
[2] U. Heinz and M. Jacob, nucl-th/0002042.

http://press.web.cern.ch/press-releases/2000/02/new-state-matter-created-cern

[3] Workshop on Future Relativistic Heavy-Ion Experiments, R. Bock, R. Stock, eds., GSI 81-6, Darmstadt (1981).

[4] E. Fermi, Prog. Theor. Physics 5570 (1950).

[5] I. Pomeranchuk, Proceedings of USSR Academy of Sciences 43889 (1951).

[6] R. Hagerdon, Nuovo Cimento Suppl. iii 2 147-186 (1965).

[7] R. Hagerdon, Nuovo Cimento Suppl. vi 2 311-354 (1968).

[8] J.C. Collins and J. Perry, Phys. Rev. Lett. 341353 (1975).

[9] N. Cabibbo and G. Parisi, Phys. Lett. B 59 67-69 (1975).

[10] N. Angert et al., Cern heavy-ion facility design Report, CERN-93-01

[11] T. Ericson, M. Jacob, H. Satz, W. Willis,. Quark Matter formation and heavy ion collisions: a workshop held at Bielefeld, Summary report prepared by the organizing committee (1982).

[12] M.G. Albrow,Workshop on SPS Fixed-Target Physics in the Years 1984-1989, ed. I. Mannelli, CERN Yellow rep. 83-02, Vol. 2, 462 (1983).

[13] E. Quercigh and K. Šafařik, Ion physics at the SPS: the experimental challenge, Physics Reports 403-404 (2004) 51-56

[14] M. Gaździcki, Recent results and plans of NA61/SHINE ,theese proceedings.

[15] I.G. Bearden et al. (NA44 Collaboration), Eur. Phys. J. C 18 317-325 (2000).

[16] H. Appelshäuser et al. (NA49 Collaboration), Eur.Phys.J. C2 (1998) 661-670 (1998).

[17] H. Appelshäuser et al. (NA49 Collaboration), Phys. Rev. Lett. 804136 (1998).

[18] T. Matsui and H. Satz, Phys. Lett. B 178416 (1986).

[19] J. Rafelski and B. Müller, Phys. Rev. Lett. 481066 (1982); ibidem Phys. Rev. Lett. 562334 (1986).

[20] B. Alessandro et al. (NA50 Collaboration), Eur. Phys. J. C 39 335-345 (2005)

[21] F. Antinori et al. (NA57 Collaboration), J. Phys. G: Nucl. Part. Phys. 32 427-441 (2006).

[22] G. Agakichiev et al. (CERES and TAPS Collaborations), Eur.Phys.J. C 4, 231 and 249 (1998).

[23] G. Agakichiev et al. (CERES Collaboration), Phys.Rev.Lett. 751272 (1995).

[24] G. Agakichiev et al. (CERES Collaboration), Phys. Lett. B 422 405-412 (1998).

[25] H.J. Specht, Nucl. Phys. A 805338 (2008).

[26] R. Arnaldi et al. (NA60 Collaboration), Eur. Phys. J. C 59 607-623 (2009).

H.J. Specht et al. (NA60 Collaboration), AIP Conf.Proc. 1322 1-10 (2010); arXiv:1011.0615.

[27] B. Abelev et al. (ALICE Collaboration), JHEP 09112 (2012).

[28] B. Abelev et al. (ALICE Collaboration), Phys. Lett. B 720 52-62 (2013).

[29] S. Chatrchyan et al. (CMS Collaboration), JHEP 05063 (2012) [arXiv:1201.5069].

[30] B. Abelev et al. (ALICE Collaboration), Phys. Rev. Lett. 111102301 (2013).

[31] B. Abelev et al. (ALICE Collaboration), Phys. Lett. B 719 18-28 (2013).

[32] S. Digal, P. Petreczky and H. Satz, Phys. Rev. D 64094015 (2001).

[33] S. Chatrchyan et al. (CMS Collaboration), Phys. Rev. Lett. 109222301 (2012).

[34] B. Abelev et al. (ALICE Collaboration), Phys. Lett. B 734 314-327 (2014).

[35] B. Abelev et al. (ALICE Collaboration), Phys. Rev. Lett. 111162301 (2013).

[36] P. Braun-Munzinger and J. Stachel, Phys. Lett. B 490196 (2000).

A. Andronic, P. Braun-Munzinger, K. Redlich and J. Stachel, Nucl. Phys. A 789334 (2007).

[37] X. Zhao and R. Rapp, Nucl. Phys. A 859114 (2011). 
[38] Y.-P. Liu, Z. Qu, N. Xu and P. Zhuang, Phys. Lett. B 67872 (2009).

[39] A Adare et al. (PHENIX Collaboration), Phys. Rev C 84054912 (2011).

[40] B. Abelev et al (ALICE Collaboration), CERNLHCC-2012-012, LHCC-I-022 (2012).

[41] B. Abelev et al. (ALICE Collaboration), J. Phys. G 41087002 (2014).

[42] G. Usai, theese proceedings.

[43] F. Fleuret, CHIC (Charm in Heavy Ion Collisions): An experiment to measure charm production at the CERN SPS, PoS(DIS 2013)252 (2013)

[44] S.J. Brodsky, F. Fleuret, C. Hadjidakis and J.P. Lansberg, Physics Reports 522 239-255 (2013).

[45] M. Koratzinos, these proceedings.

[46] N. Armesto et al., in the proceedings of the 2014 Quark Matter conference, arXiv:1407.7649. 